\title{
Evacuation Guidance Design: An Experimental Study Based on Eye Tracking Devices
}

\author{
Ning Ding ${ }^{1}$, Tao Chen ${ }^{2}$, Yuan Liu ${ }^{3}$ \\ ${ }^{1}$ School of Criminal Investigation and Counter-Terrorism \\ People's Public Security University of China, Beijing, China \\ dingning_thu@126.com \\ ${ }^{2}$ Institute of Public Safety Research, Department of Engineering Physics \\ Tsinghua University, Beijing, China \\ chentao.a@tsinghua.edu.cn \\ ${ }^{3}$ Institute of Economics, School of Social Sciences \\ Tsinghua University, Beijing, China \\ liuyuan_thu@163.com
}

\begin{abstract}
It is a crucial issue whether evacuees follow the evacuation guidance during evacuation. Good evacuation guidance is necessary to be designed to help the evacuees follow the guidance. In this paper, evacuation experiments based on wearable eye tracking devices were carried out to study the design effect of the evacuation guidance. Three factors were considered in these experiments: 1) the position of the evacuation guidance; 2) follow guidance or follow other evacuees; 3 ) follow a stranger or a familiar person. The results show that more participants noticed the guidance with low position and ground position than the guidance with up position. The evacuees intend to follow others rather than to follow the guidance, i.e. most evacuees act as "follower". Eye tracking evacuation experiments can also be used to test the effectiveness of evacuation guidance signs.
\end{abstract}

Keywords: evacuation guidance; experiments; eye tracking devices; leader-and-follower

\section{Introduction}

With the continuous development of modern architecture, both the structure and the function of a building are gradually becoming more complicated. Thus, evacuation of people under emergency circumstances becomes an important research topic nowadays. Complex indoor places are usually enclosed environments with internal obstacles and multiple exits. They have features such as large scene area, complex internal structures, and multiple exits, such as supermarkets, movie theaters, and gymnasiums. Unlike pedestrian evacuation modeling in a simple environment (access, barrier-free rooms) whose focus is on research at the operational level, pedestrian evacuation modeling in complex indoor places needs to consider behavioral characteristics at the tactical level ${ }^{[1]}$. How do evacuees choose the route in the evacuation process? How to make a decision? Do they follow the evacuation guidance? Only solving the problems of evacuation behaviors can provide a strong basis for the design of building evacuation signs and the establishment of evacuation simulation models.

In general, it is necessary to conduct evacuation experiments to study the crowds' evacuation behavior. The reason is that the evacuation data in fire disasters cannot be collected or the collection is incomplete, and we cannot ignite a building to conduct evacuation experiments. The experiment to study the crowds' evacuation behavior is divided into five types: (1) global evacuation drills ${ }^{[2]}{ }^{[5]}$; (2) local evacuation experiments ${ }^{[6]-[9] ;}$; 3 ) controlled pedestrian flow experiments ${ }^{[10]-[13]}$; (4) computer simulation evacuation experiments ${ }^{[14]-[18]}$; (5) virtual reality experiments ${ }^{[19]-[22]}$. Other research methods include the use of animals and insects for evacuation studies ${ }^{[23]}$, and in particular research on evacuation in panic situations [24] - [25]. However, these experimental studies mainly focused on the microscopic and macroscopic characteristics of pedestrian flow, and they did not fully grasp the decision-making process of the crowd. In 
order to further analyze the evacuees' psychology and behavior, eye tracking devices can be introduced in evacuation experiments.

Eyes are an important way for humans to obtain information. They can reflect people's psychological activities to some extent. The eye movement technique is to extract the data such as the gaze point, the gaze time, the number of gazes, the saccade distance, and the pupil size from the records of the eye movement trajectory, so as to study the intrinsic cognitive process of the individual. In recent years, eye tracking devices have also received some attention in evacuation experiments. Cosma ${ }^{[26]}$ combined VR technology with eye tracker to build a smoke-filled railway tunnel evacuations scene. A green LED lighting system was installed on the road surface. The crowd path selection behavior under different lighting conditions were studied through virtual reality evacuation experiments. It was found that the lighting system has a significant positive impact on the crowd's safety evacuation. Andree ${ }^{[27]}$ built a virtual high-rise building through VR technology. Through experiments, they studied the export selection behavior of crowds during the evacuation process and the waiting time when using elevators for evacuation. It was found that green evacuation indicators can effectively guide evacuees to choose elevators. However, there are still few experimental studies using eye trackers in evacuations.

In this paper, experiments were carried out to study how the position of the evacuation guidance sign influences evacuees' way-finding behaviors, and the impact of leader-and-follower on the way-finding choices during evacuation. In order to study these issues, we carried out 7 experiments with the use of wearable eye tracking glasses. The building layout and the procedure of the experiments are introduced in Section 2. The results of these experiments, including the ratio of different way-finding choices and the fix-action, are shown in Section 3. In the Section 4, the comparison of these results and some interesting phenomena during the experiments are discussed.

\section{Experiment}

In this paper, 7 experiments were carried out in a complex building. The objectives of these experiments are: (1) the impact of the positions (high, low, ground) of the evacuation guidance sign on the way-finding behavior; (2) when facing to a guidance sign and another evacuee (a stranger or an acquaintance), how does the participant make his/her choice? (3) when facing to an acquaintance and a stranger, the participant tends to follow which person?

The experiments were carried out in a complex building in People's Public Security University of China. This building is used for police and student training, and there are several rooms, crossroads and staircases. The Room 8 on second floor was used for the experiments, and the layout of the second floor is shown in Fig.1. When a participant arrived at this floor, he/she was blindfolded, and an experimenter guided him/her to a room. Then the eye mask was removed, and the experimenter helped him/her wear the eye tracking glasses (the device is SMI ETGTM). The eye tracking glasses is shown in Fig.2(a), and this glasses is connected with a mobile phone (shown in Fig.2(b)), which is used to storage the experimental data. Participant can easily wear the glasses with the mobile phone in his/her pocket. After calibrating the eye tracking glasses, the participant was asked to stand back to the door with 3 meters. When he/she hears the alarm sound, the experiment is started immediately, and when the evacuee (Participant A) finds the stairwell, the experiment is terminated. In an experiment involving other evacuees (Participant B), participant B stood near the door but did not obstruct the sight of participant A. In order not to introduce interference to the experiments, participant $\mathrm{B}$ and participant $\mathrm{C}$ were asked to wear the same clothes and pants. 


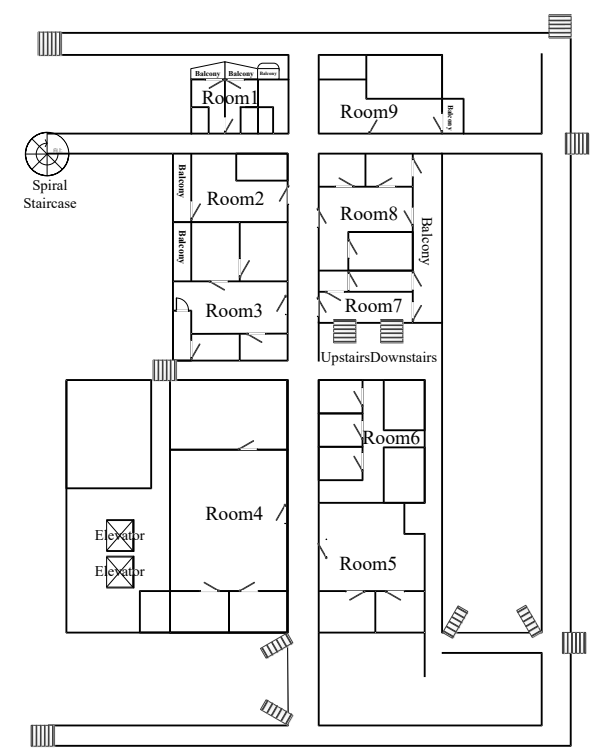

Fig. 1: Layout of the second floor.

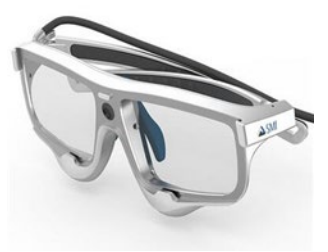

a

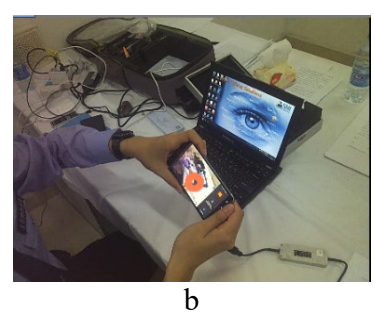

Fig.2 Eye tracking devices.

Before the experiment begins, the participant is asked if he knows which room he is in. If he knows the room, the experiment is considered invalid. The subjects of this experiment were all undergraduates of the School of Criminal Investigation and Counter-Terrorism, People's Public Security University of China. Each experiment includes 50 volunteers, and a total of 300 students participated in the 6 groups of experiments.

The experiment procedure is as follows:

1) Experiment 1: the guidance sign is set $30 \mathrm{~cm}$ higher than the ground on the wall.

2) Experiment 2: the guidance sign is set $200 \mathrm{~cm}$ higher than the ground on the wall.

3) Experiment 3: the guidance sign is set right ahead of the door threshold on the ground.

4) Experiment 4: the guidance sign is set $30 \mathrm{~cm}$ higher than the ground on the wall, and a stranger stood around the door inside the room. The stranger was asked to go to the opposite direction of the guidance sign.

5) Experiment 5: the guidance sign is set right ahead of the door threshold on the ground, and a stranger stood around the door inside the room. The stranger was asked to go to the opposite direction of the guidance sign.

6) Experiment 6: there is no guidance sign at the door. The stranger (Participant $\mathrm{B}$ ) and a person (participant C) familiar with the evacuee (Participant A) waited in the room before the experiment. When the experiment started, B moved to the left, and C moved to the right. Participant A was behind $\mathrm{B}$ and $\mathrm{C}$ before the evacuation began.

\section{Results}

\subsection{Analysis Standard}

Eye tracking devices are used during evacuation. Because the background is a dynamic picture, the analysis method is different from that for static pictures. This experiment is mainly to test the relationship 
between the way-finding behaviour and eye movement in the evacuation process. In general, in experiment 1-3, eye movement characteristics of evacuees during evacuation can be divided into two categories: 1) the evacuee did not look at the guidance sign; 2) the evacuee looked at the guidance sign. Then the evacuees who looked at the guidance signs can be divided into people following the guidance sign and people not following the guidance sign. In experiment 4-5, the eye movement characteristics during evacuation can be divided into four categories: 1) the evacuee only looked at the guidance sign; 2) the evacuee only looked at participant B; 3) the evacuee looked at the guidance sign and also looked at participant B; 4) the evacuee did not look at the guidance sign, and did not look at participant B. The fourth category of evacuees make their own decision without the influence of the sign or participate B, so there is no need to analyse whether their evacuation direction is in accordance with the direction the sign indicated. Category 1-3 will be analyzed later.

In Experiment 6, the eye movement characteristics during evacuation can also be divided into four categories: 1) evacuee only looked at participant B; 2) evacuee only looked at participant C; 3) evacuee looked at participant $\mathrm{B}$ and also looked at participant $\mathrm{C}$; 4) evacuee did not look at participant $\mathrm{B}$, and also did not look participant C. Similar to the analysis of experiment 4-5, the fourth category is the independent decision of the evacuee. There is no need to analyze whether the evacuee follows the participant $\mathrm{B}$ or the participant $\mathrm{C}$, and the other three categories need further analysis.

The data of the eye tracker mainly analyzes the time when evacuee looks at the sign: 1) if evacuee looks at the guidance sign, the time starts to count from when the evacuee's gaze falls on the sign, and stops when the gaze point is removed from the sign; 2) if evacuee looks at other participant, when evacuee begins to follow a stranger or opinion leader, the timing begins. When the gaze point moves away from the other participant and turns to another place (such as an intersection) during the evacuation process, the timing stops.

In the course of the experiment, some of the participants' eye movement data was invalid, mainly due to the fact that the eyeglasses were loose during the evacuation process and the fix-action was inaccurate. Another reason is that they mentioned previously that they knew the location of the room they were in.

\subsection{Results of Experiments 1-3}

A snapshot of experiment 1 is shown in Fig.3, and Table 1 shows the results of experiment 1-3. For the lower, upper, and ground signs, the percentage of people who could see the sign is $42.55 \%, 16.67 \%$, and $47.73 \%$, respectively. The guidance signs used in this experiment are evacuation indicators without light-assisted lighting. It can be seen from the experimental results, the percentage of evacuees who can see evacuation guidance sign is less than $50 \%$, where the sign in lower and ground position is more likely to attract evacuees' attention than signs in upper position.

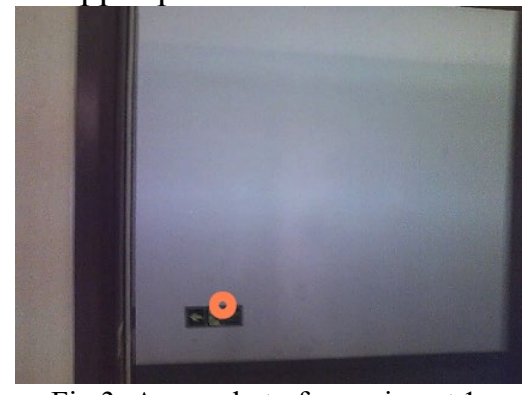

Fig.3: A snapshot of experiment 1.

Table 1. Results of experiment 1-3.

\begin{tabular}{|c|c|c|c|c|c|c|c|c|c|}
\hline Experiment & $\begin{array}{c}\text { Number of } \\
\text { valid } \\
\text { participants }\end{array}$ & $\begin{array}{c}\text { Sign } \\
\text { position }\end{array}$ & $\begin{array}{c}\text { Number of } \\
\text { participants } \\
\text { who see the } \\
\text { sign }\end{array}$ & Follower & $\begin{array}{c}\text { Average } \\
\text { gaze } \\
\text { time }\end{array}$ & Non-follower & $\begin{array}{c}\text { Average } \\
\text { gaze } \\
\text { time (s) }\end{array}$ & $\begin{array}{c}\text { Follow-then-backtrack } \\
\text { Average } \\
\text { gaze } \\
\text { time }\end{array}$ \\
\hline 1 & 47 & Low & 20 & 12 & 0.54 & 6 & 1.04 & 2 & 0.48 \\
\hline 2 & 42 & High & 7 & 5 & 0.53 & 2 & 0.52 & 0 \\
\hline 3 & 44 & Ground & 21 & 14 & 0.61 & 6 & 0.42 & 1 & - \\
\hline
\end{tabular}


Among people who saw the guidance sign, $60.00 \%, 71.43 \%$ and $66.67 \%$ chose to follow the sign, respectively. It can be seen that $60 \%-70 \%$ of those who saw the sign chose to follow direction the sign indicates. There are also individual participants who chose to follow the evacuation signs to evacuate and then went back to the other direction. In terms of the gazing time of the evacuation guidance sign, the average time of the follower in experiment 1 was only half that of the non-follower. However, in experiment 3, the follower's average gaze time is longer than that of the non-follower. It can be seen that there is no significant feature in the gaze time.

\subsection{Results of Experiments 4-5}

Snapshots of experiment 4 are shown in Fig.4, and Table 2 shows the results of experiment 4-5. In experiment 4-5, most evacuees only saw the evacuation guidance sign or participant B. In both sets of experiments, most of the people who only saw the sign and only saw participant B would choose to follow the directions of the sign or participant B respectively (depending on what they saw). Only in experiment 5, although 2 evacuees only saw the evacuation guidance sign, they did not follow the direction the sign indicated. Among the evacuees who saw the sign, the proportion of evacuees following the signs in experiments 4 and 5 was significantly higher than that in experiment 1-3.
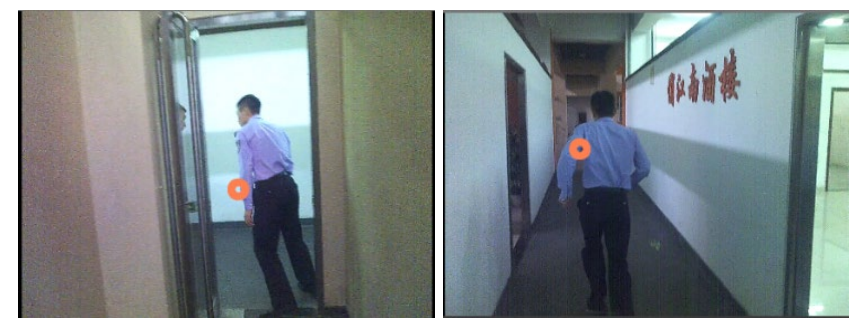

Fig.4: Snapshots of experiment 4.

Table 2. Results of experiment 4-5.

\begin{tabular}{|c|c|c|c|c|c|}
\hline \multicolumn{6}{|c|}{ Experiment 4,36 valid data 36 persons, sign position: low } \\
\hline Category & Persons & $\begin{array}{c}\text { Persons } \\
\text { following the } \\
\text { sign }\end{array}$ & $\begin{array}{l}\text { Average gaze time } \\
\quad \text { (seconds) }\end{array}$ & $\begin{array}{l}\text { Persons following } \\
\text { participant B }\end{array}$ & $\begin{array}{l}\text { Average gaze time } \\
\quad \text { (seconds) }\end{array}$ \\
\hline Only saw sign & 10 & 10 & 0.74 & 0 & - \\
\hline $\begin{array}{c}\text { Only saw participant } \\
\text { B }\end{array}$ & 15 & 0 & - & 15 & 3.75 \\
\hline Saw both & 6 & 6 & 0.66 & 0 & 3.58 \\
\hline Saw now & 5 & - & - & - & - \\
\hline \multicolumn{6}{|c|}{ Experiment 5 , valid data 43 persons, sign position: ground } \\
\hline Category & Persons & $\begin{array}{c}\text { Persons } \\
\text { following the } \\
\text { sign }\end{array}$ & $\begin{array}{l}\text { Average gaze time } \\
\quad \text { (seconds) }\end{array}$ & $\begin{array}{l}\text { Persons following } \\
\text { participant B }\end{array}$ & $\begin{array}{l}\text { Average gaze time } \\
\quad \text { (seconds) }\end{array}$ \\
\hline Only saw sign & 10 & 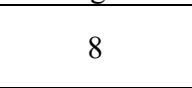 & 0.77 & 2 & $\begin{array}{c}0.79 \text { (time gazing at the } \\
\text { signs) }\end{array}$ \\
\hline $\begin{array}{c}\text { Only saw participant } \\
\text { B }\end{array}$ & 18 & 0 & - & 18 & 2.88 \\
\hline Saw both & 2 & 1 & 0.66 & 1 & 0.61 \\
\hline Saw now & 13 & - & - & - & - \\
\hline
\end{tabular}

In both sets of experiments, the number of persons who only saw and then followed participant B was significantly higher than the number of people who only saw evacuation signs and followed. The average time of the gaze ( 3.75 seconds and 2.88 seconds) was much higher than that of participants who only saw the evacuation signs ( 0.74 second and 0.77 second). The reason is mainly because when evacuees follow other people for evacuation, they will follow this person and watch for a long time.

In experiment 4, six participants saw both the evacuation guidance sign and participant $\mathrm{B}$, but they all chose to follow the direction the sign indicated. It can be seen from their time of fixation that they look at 
participant B much longer than when they watch the evacuation sign. In experiment 5 , due to the small sample size, there were no significant features.

\subsection{Results of Experiments 6}

Table 3 shows the results of experiment 6 . In experiment 6 , the number of evacuees who only saw participant B (stranger) or participant $\mathrm{C}$ (acquaintance) are close. And there is no significant difference between the gaze time between the two categories. Of the 15 evacuees who both saw participant $\mathrm{B}$ and $\mathrm{C}, 7$ of them chose to follow participant B (stranger), and 8 chose to follow acquaintance. In terms of gaze time, the time to gaze an acquaintance is obviously greater than the time to look at strangers. In addition, there were four people who did not look at familiar people or strangers and made their choices based on their own judgment.

Table 3. Results of experiment 6.

\begin{tabular}{|c|c|c|c|c|c|}
\hline \multicolumn{7}{|c|}{ Experiment 6, valid data 6 persons } \\
\hline Category & Persons & $\begin{array}{c}\text { Persons following } \\
\text { participant B }\end{array}$ & $\begin{array}{c}\text { Average gaze time } \\
\text { (seconds) }\end{array}$ & $\begin{array}{c}\text { Persons following } \\
\text { participant C }\end{array}$ & $\begin{array}{c}\text { Average gaze time } \\
\text { (seconds) }\end{array}$ \\
\hline $\begin{array}{c}\text { Only saw } \\
\text { participant B }\end{array}$ & 14 & 13 & 3.02 & 1 & $\begin{array}{c}0.7 \text { (the time gazing } \\
\text { at B) }\end{array}$ \\
\hline $\begin{array}{c}\text { Only saw } \\
\text { participant C }\end{array}$ & 13 & 2 & $\begin{array}{c}0.43 \text { (the time gazing at } \\
\text { participant C) }\end{array}$ & 11 & 3.76 \\
\hline Saw both & 15 & 7 & $\begin{array}{c}\text { participant B: } 2.01 \\
\text { participant C: } 2.43\end{array}$ & 8 & $\begin{array}{c}\text { participant B: } 1.70 \\
\text { participant C: } 3.09\end{array}$ \\
\hline Saw now & 4 & - & - & - & - \\
\hline
\end{tabular}

\section{Comparison and Discussion}

\subsection{Comparison}

As stated earlier, in experiments 1 and 4, the guidance sign is set $30 \mathrm{~cm}$ higher than the ground on the wall, in experiment 2 is set $200 \mathrm{~cm}$ higher than the ground on the wall and in experiment 3 and 5 right ahead of the door threshold on the ground. By comparing the proportions of evacuees who gazed at the guidance sign in experiments 1-5, as shown in Fig.5, results indicate that it is harder to be noticed when the position of the sign is higher. And only $16.67 \%$ of evacuees noticed the guidance sign set $200 \mathrm{~cm}$ above the ground which is the highest sign position in all the six experiments. In contrast, the effect of an evacuation sign set in a lower position is relatively good. However, the evacuation signs used in this experiment were not light-assisted. In other VR evacuation experiments, it was found that the green LED evacuation guidance signs had good effect. In future experiments, we will also consider light-assisted evacuation indicators ${ }^{[26,27]}$.

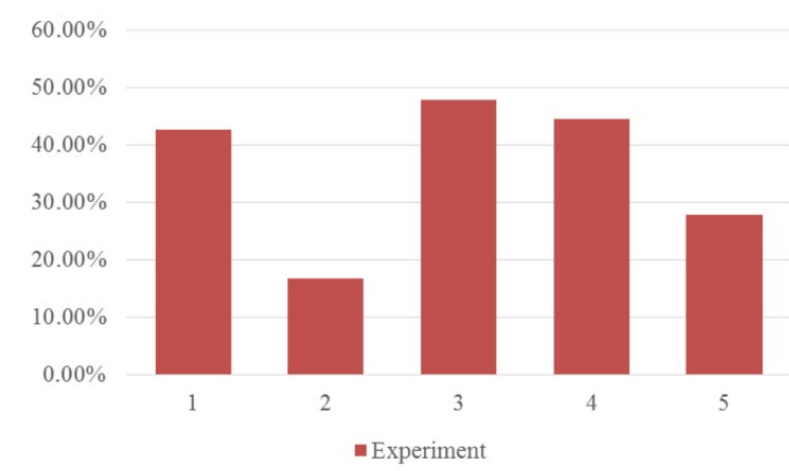

Fig.5 Proportions of evacuees who saw guidance sign in experiment 1-6.

The evacuees' average gaze time in experiment 1-6 was as shown in Fig.6. In all the 6 experiments the duration of gazing at the guidance sign was relatively short, concentrating between $0.5 \mathrm{~s}$ and $1 \mathrm{~s}$. In contrast, the duration of gazing at people is longer because evacuees who follow people kept gazing at them during the evacuation. In experiment 6 , the time duration of gazing at acquaintances is longer than at strangers. 


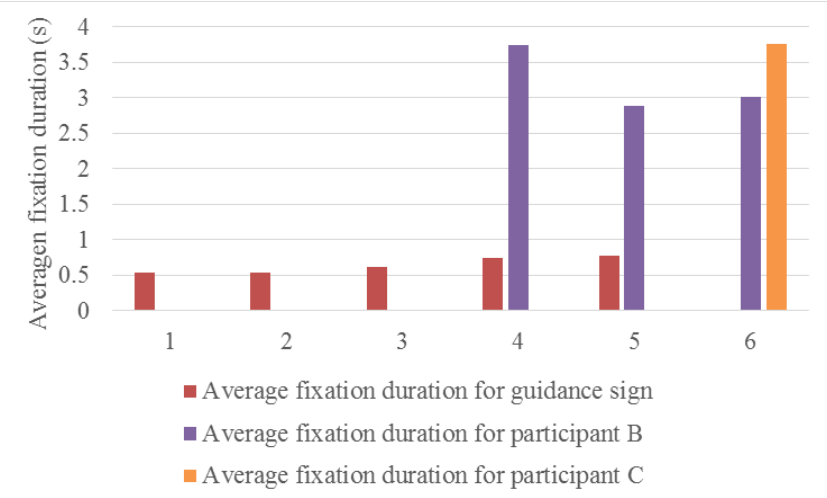

Fig.6 Average gaze time of the evacuees in experiment 1-6.

In the experiment, the proportion of evacuees who only saw the evacuation guidance sign or only saw another participant and acted as follower is shown in Fig.7. It shows that among the evacuees who only saw the guidance sign or another participant, the proportion of choosing to follow another participant is higher than that to follow the signs. By comparing experiments 1 and 4, experiments 3 and 5, it was found that although the location of the evacuation sign was the same, the proportion of evacuees who chose to follow the evacuation sign is higher when participant $\mathrm{B}$ is involved in the experiment. In addition, in the experiment 4-6, participants have a higher probability of following the target (guidance sign or other participants) they saw, and in experiments 4 and 5, the percentage of following strangers is $100 \%$. In the experiment, directions of the sign and participant B are opposite. If the directions are the same, the probability that the crowd will follow the guidance sign may increase, and further studies will be conducted in later experiments.

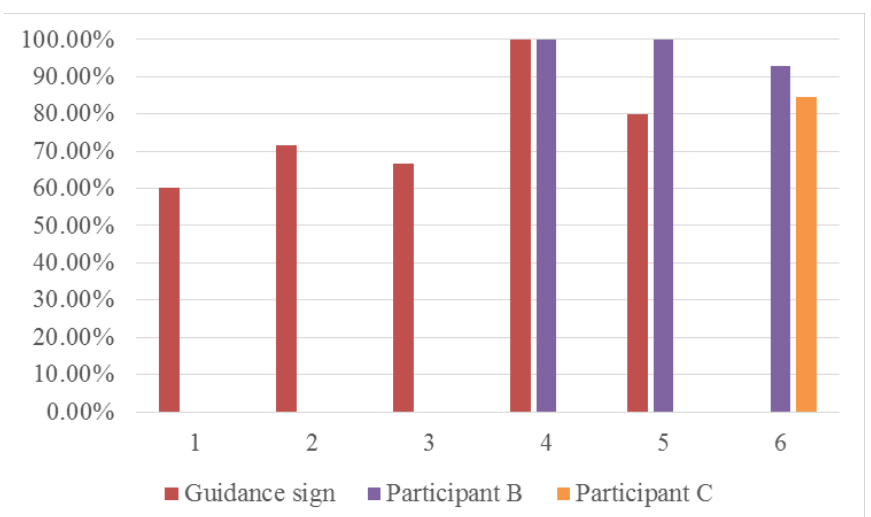

Fig.7 The proportion of evacuees following the target they saw.

In Fig. 8 we compare the number of evacuees who only gazed at evacuation guidance signs and at other participants in experiment 4 and experiment 5 . The number of evacuees watching strangers was higher than the number of evacuees watching the evacuation signs. It can be seen that in the process of evacuation, the attraction of the other people is greater than the attraction of the guidance signs to the evacuees. 


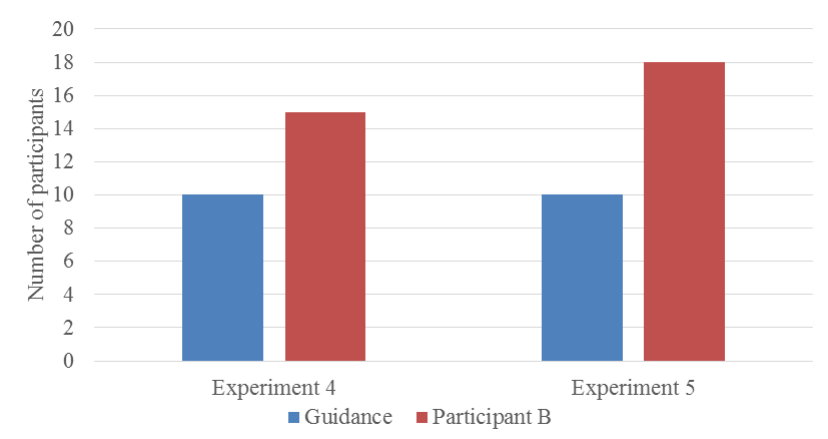

Fig.8. Number of evacuees who only saw the sign or other participants.

\subsection{Limitations}

There is a gap between experiments and real emergency evacuation events. The participants in our experiments were all Chinese students and well-educated; the type of participants and Chinese culture may have impact on the experimental results.

\section{Conclusion}

In this paper, 6 experiments were carried out. According to the results of experiments, it is found that more participants noticed the guidance with low position and ground position than the guidance with up position. The evacuees intend to follow others than to follow the guidance. Most of the evacuees play the role of a "follower". Eye tracking evacuation experiments can be used to test the effectiveness of evacuation guidance signs. In our future work, more features should be tested based on experiments, such as social influence or social bond, guidance sign with lighting.

\section{Acknowledgements}

This work is supported by National Key R\&D Program of China (No. 2017YFC0803300), the National Natural Science Foundation of China (71373139, 91646201).

\section{References}

[1] Hoogendoorn, S., and W. Daamen. "Self-organization in pedestrian flow." Traffic and Granular Flow 2003:373-382.

[2] Peacock, R. D., B. L. Hoskins, and E. D. Kuligowski. "Overall and local movement speeds during fire drill evacuations in buildings up to 31 stories." Safety Science, vol. 50, no. 8, pp. 1655-1664, 2012.

[3] Huo, F., et al. "Experimental study on characteristics of pedestrian evacuation on stairs in a high-rise building." Safety Science, vol. 86, pp. 165-173, 2016.

[4] Cheng, X., et al. "Study of announced evacuation drill from a retail store." Building \& Environment, vol. 44, no. 5, pp. 864-870, 2009.

[5] Kobes, M., et al. "Building safety and human behaviour in fire: A literature review." Fire Safety Journal, vol. 45, no. 1, pp. 1-11, 2010.

[6] Ma, J., et al. "Experimental study on an ultra high-rise building evacuation in China." Safety Science, vol. 50, no. 8, pp. 1665-1674, 2012.

[7] Fang, Z., et al. "Experiment and modeling of exit-selecting behaviors during a building evacuation." Physica A Statistical Mechanics \& Its Applications, vol. 389, no. 4, pp. 815-824,2010.

[8] Fang, Z., et al. "Experimental study on evacuation process in a stairwell of a high-rise building." Building \& Environment, vol. 47, no.1, pp. 316-321, 2012.

[9] Liao, Y. J., et al. "A Study on People's Attitude to the Use of Elevators for Fire Escape." Fire Technology, vol. 50, no. 2, pp. 363-378, 2014.

[10] Isobe, M., T. Adachi, and T. Nagatani. "Experiment and simulation of pedestrian counter flow." Physica A Statistical Mechanics \& Its Applications, vol. 336, no. 3, pp. 638-650, 2004. 
[11] Kretz, T., A. Grünebohm, and M. Schreckenberg. "Experimental study of pedestrian flow through a bottleneck." J.Stat.Mech. (2006) P10014.

[12] Seyfried, A., et al. "New Insights into Pedestrian Flow Through Bottlenecks."Transportation Science, vol. 43, no. 3, pp. 395-406, 2009.

[13] Zhang J, W. Klingsch, A. Schadschneider, et al. "Experimental study of pedestrian flow through a T-junction." Traffic and Granular Flow 11. Springer Berlin Heidelberg, 2013: 241-249.

[14] Liu, S. B., et al. "Modeling Movement Direction Choice and Collision Avoidance in Agent-Based Model for Pedestrian Flow." Journal of Transportation Engineering, vol. 141, no. 6, pp. 1-8, 2015.

[15] Wei, J., et al. "Experiment of bi-direction pedestrian flow with three-dimensional cellular automata." Physics Letters A, vol. 379, no. 16-17, pp. 1081-1086, 2015.

[16] Isobe, Motoshige, T. Adachi, and T. Nagatani. "Experiment and simulation of pedestrian counter flow." Physica A Statistical Mechanics \& Its Applications, vol. 336, no. 3, pp. 638-650, 2004.

[17] Helbing, D., et al. "Self-Organized Pedestrian Crowd Dynamics: Experiments, Simulations, and Design Solutions." Transportation Science, vol. 39, no. 1, pp. 1-24, 2005.

[18] Hu, J., et al. "Experiment and simulation of the bidirectional pedestrian flow model with overtaking and herding behavior." International Journal of Modern Physics C, vol. 26, no. 11, pp. 1-12, 2015.

[19] Kinateder M., E. Ronchi, D. Nilsson, et al., "Virtual reality for fire evacuation research." IEEE Federated Conference on Computer Science and Information Systems (FedCSIS), 2014, pp. 313-321.

[20] Ronchi, E., et al. "A Virtual Reality Experiment on Flashing Lights at Emergency Exit Portals for Road Tunnel Evacuation." Fire Technology, vol. 52, no. 3, pp. 623-647.

[21] Marsh K L, Wilkie C T, Luh P B, et al. "Crowd Guidance in Building Emergencies: Using Virtual Reality Experiments to Confirm Macroscopic Mathematical Modeling of Psychological Variables." Pedestrian and Evacuation Dynamics 2012. Springer International Publishing, 2014: 197-212.

[22] Lu X., P.B. Luh, K.L. Marsh, et al. "Guidance optimization of building evacuation considering psychological features in route choice." Intelligent Control and Automation (WCICA), 2014 11th World Congress on. IEEE, 2014: 2669-2674.

[23] Lin P., J. Ma, T. Liu, et al. "An experimental study of the "faster-is-slower" effect using mice under panic." Physica A Statistical Mechanics \& Its Applications, vol. 452, pp. 157-166, 2016.

[24] Shiwakoti, N., and M. Sarvi. "Enhancing the panic escape of crowd through architectural design." Transportation Research Part C, vol. 37, no. 3, pp. 260-267, 2013.

[25] Shiwakoti, N., et al. "Animal dynamics based approach for modeling pedestrian crowd egress under panic conditions." Transportation Research Part B 45.9(2011):1433-1449.

[26] Cosma, G., E. Ronchi, and D. Nilsson. "Way-finding lighting systems for rail tunnel evacuation: A virtual reality experiment with Oculus Rift ${ }^{\circledR}$." Journal of Transportation Safety \& Security8.sup1(2016):101-117.

[27] Andrée, K., D. Nilsson, and J. Eriksson. "Evacuation experiments in a virtual reality high - rise building: exit choice and waiting time for evacuation elevators." Fire \& Materials 40.4(2016):554-567. 